\title{
Mesenchymal stem cells: applications in immuno-cell therapy
}

Nasim Rahmani Kukia', Payam Zandi ${ }^{2}$, Ardeshir Abbasi ${ }^{3 *}$

'Department of Microbiology, Faculty of Veterinary Medicine, Urmia University, Urmia, Iran

${ }^{2}$ Department of Medical Biotechnology, Faculty of Medical Sciences, Tarbiat Modares University Tehran, Iran

${ }^{3}$ Department of Immunology, Faculty of Medical Sciences, Tarbiat Modares University, Tehran, Iran

Article Info

\section{Article Notes}

Received: June 04, 2018

Accepted: July 05, 2018

\section{${ }^{*}$ Correspondence:}

Dr. Ardeshir Abbasi, Department of Immunology, Faculty of Medical Sciences, Tarbiat Modares University, Tehran, Iran; Telephone No: +989187262126;

Email: ardeshir.abbasi66@gmail.com

C) 2018 Abbasi A. This article is distributed under the terms of the Creative Commons Attribution 4.0 International License.

\section{Keywords}

Mesenchymal stromal cell, Macrophage

Neutrophil

Nicotine, Caffeine

Vitamin D3

\section{ABSTRACT}

Mesenchymal stromal cells(MSCs) have been exploited for their immunomodulatory properties in treating various immune-related disorders. MSCs can modulate the immune system through interactions with a variety of immune cells. Regardless of the researchers focused on understanding how MSCs connect to individual immune system cell subsets, the mechanisms for inducing restorative effect still stay mainly undiscovered. Through this mini-review we address what is known about the associations and effects of educated MSCs with cells of the innate immune system (macrophages and neutrophils) and our knowledge of these interactions will be essential in increasing and expanding new medical protocols for MSC based cell therapy in the foreseeable future

\section{Introduction}

Mesenchymal stem (or stromal) cells (MSCs) are fibroblastlike, multipotent progenitor cells which can be isolated from bone marrow, umbilical cord, gingiva, fats, and other tissues ${ }^{1}$. MSCs also have powerful immunomodulatory properties and can be considered as a potential tool for cell-based immunotherapy ${ }^{2}$. There is a strong connection between the MSCs in bone marrow and hematopoietic cells including macrophages and neutrophils. MSCs are located in the periendothelial area, therefore they collaborate with blood cells, such as neutrophils and macrophages especially during an inflammatory state ${ }^{3}$. Macrophages are fundamental effectors and modulator cells in orchestrating the innate and adaptive immune system responses ${ }^{4}$. In addition, neutrophils are the first and most important cells of the innate immune system in response to infections and irritation ${ }^{5}$. MSCs have been reported to show immunomodulatory effects on both humoral and cellular immunity and also on different elements of the innate immune system. This mini-review has focused on the effects of MSC on the phenotypic shift of neutrophils and macrophages.

\section{Mesenchymal stem cells (MSCs)}

MSCs are plastic, fibroblast-like, adherent, multipotent nonhematopoietic progenitor cells that can mature into like bone, cartilage, and fat cells ${ }^{6}$. MSCs express various number of markers but none of them are specific for them. In adults, MSCs lack hematopoietic markers, such as CD45, CD34, CD11 and CD14. They express adhesion molecules, such as CD160 and CD106, CD29, ICAM1 and other markers, such as CD73, CD90, CD44 and CD105. However, they do not express costimulatory molecules such CD86, 
CD80 and CD40. An advantage of transplanting MSCs is that, because of their non-immunogenic phenotype, they do not require immunosuppression. ${ }^{7}$ MSCs have been proven to own an immunoregulatory role as they interact with numerous myeloid and lymphocytic leukocytes; therefore, MSCs may be regarded as valuable tools for cellbased immunotherapy ${ }^{8}$. Nowadays, MSCs have been used especially in cell therapy projects for autoimmune and inflammatory diseases because of their role in modifying immune system along with regenerative potential that MSCs possess ${ }^{6}$. MSCs in tissues and bone marrow set up a niche which leads to an essential connection with hematopoietic cells including macrophages and neutrophils 9 . However, the nature and mechanism of this connection is not yet disclosed. With increasing data for the role of MSCs in the immediate modulation of the innate immune system, targeting MSCs as a treatment strategy is gradually being seen as a novel and attractive treatment for diseases like: bacterial infections ${ }^{10}$, severe lung damage ${ }^{11}$ and sepsis ${ }^{12}$.

\section{MSCs and Neutrophils}

Neutrophils will be the most dominant cells of the innate immune system and are predominant in various tissues during severe inflammatory responses ${ }^{9}$. Neutrophils also play the effector role in adaptive immunity ${ }^{9}$. Mature neutrophils leave the bone marrow and move toward the blood vessels and further to the inflamed areas where they can interact with the MSCs, localized in the perivascular and periendothelial areas ${ }^{3}$. Previous studies have proved that MSCs help neutrophils in their function. However, the effects of educated MSCs on neutrophils had not been made clear. Therefore, we investigated the function of neutrophils after MSCs were treated with different concentrations of nicotine and caffeine. We also examined the effect of mesenchymal inflammation on the neutrophils functions. The results of MSCs treated with different concentrations of caffeine included: an increment in phagocytosis of neutrophils in contact with MSCs treated with caffeine, significant reduction in the reactive oxygen substances compared to MSCs without treatment ${ }^{13}$.

MSCs treated with nicotine exerted a protecting role for the essential effector functions of neutrophils against an unexpected non-specific, and possibly unsafe reactive oxygen species (ROS). ROS is a chemical compound that mounts an attacking response, in neutrophils and macrophages, in order to kill pathogens. Nevertheless, high levels of nicotine as in smokers, can hinder some effects of MSCs on neutrophils and reduce the capability of neutrophils to remove pathogens ${ }^{14}$. Moreover, in the final investigation, it was seen that when the LPS-primed MSCs were treated with nicotine or caffeine, the vitality of co-cultured neutrophils was significantly increased. Lipopolysaccharide, LPS, is a building block of the membrane of gram negative bacteria. This fever-inducing substance is considered as a strong antigen that binds to toll-like receptors and triggers an immune response against the pathogens that comprise them. Hence, LPS were an excellent choice for stimulating pro-inflammation of MSCs ${ }^{15}$. The rate of the respiratory burst of neutrophils after being co-cultured with LPS-primed MSCs reduced compared to the respiratory burst of neutrophils by them self ${ }^{16}$. Generally, these findings might provide a new insight into understanding the anti-inflammatory and immunomodulatory effects of nicotine and caffeine.

\section{MSCs and Macrophages}

Macrophages have critical effector functions and are modulator cells in the synchronizing the initiation of innate and adaptive immune system responses ${ }^{17}$. Despite their primary function to eliminate pathogens, these cells play an important role in guiding other immune system cells. They also exhibit a function in degenerative diseases ${ }^{18}$. Furthermore, macrophages have an important function in later stages of homeostasis system and tissue repair, such as removal of cellular debris and clearance of apoptotic cells. Macrophages are extremely plastic and based on the environmental factors, they can shift between phenotypes ${ }^{18}$. It is known that MSCs modulate the immune system via multiple mechanisms. For example, induction of M2 macrophages with anti-inflammatory capacity is one of the recently identified mechanisms that MSCs exert their immunomodulatory effects. In addition, when cocultured with tissue derived stem cells, macrophages showed anti-inflammatory characteristics and an increase in CD206 marker expression was seen ${ }^{19}$. The nature of the later mechanism is not yet known. Therefore, our coauthor investigated functions of macrophages after MSCs treatment with vitamin D3 and caffeine. The results of MSCs treated with caffeine included: significant increase in phagocytosis and collectively regressed concentrations of ROS and nitric oxide (NO) as well as IL-12 secreted by macrophages change the characteristics of MSCs into not educated cells ${ }^{20}$. MSCs Treated with vitamin D3 stimulate anti-inflammatory macrophages by increasing the vitality and the phagocytosis capacity of macrophages and reducing the potentially dangerous creation of ROS ${ }^{21}$. Those results might offer new conception into the potential mechanisms that underlie the immunomodulatory and anti-inflammatory effects of caffeine or vitamin D3.

\section{Conclusion}

Collectively, when treated with an agent such as nicotine, caffeine and vitamin D3, MSCs can affect the phenotype and function of the immune system cells. Further understanding of our knowledge about these interactions will be essential in increasing and expanding new medical protocols for MSC based cell therapy in the future. 


\section{References}

1. Glenn JD, Whartenby KA. Mesenchymal stem cells: emerging mechanisms of immunomodulation and therapy. World journal of stem cells. 2014; 6: 526.

2. Wada N, Gronthos S, Bartold PM. Immunomodulatory effects of stem cells. Periodontology 2000. 2013; 63: 198-216.

3. Grando F, Felicio C, Twardowschy A, et al. Modulation of peritoneal macrophage activity by the saturation state of the fatty acid moiety of phosphatidylcholine. Brazilian Journal of Medical and Biological Research. 2009; 42: 599-605.

4. Stromberg P, Guillot F. Hematology in the regressive phase of bovine psoroptic scabies. Veterinary pathology. 1987; 24: 371-377.

5. Zhang R, Liu Y, Yan K, et al. Anti-inflammatory and immunomodulatory mechanisms of mesenchymal stem cell transplantation in experimental traumatic brain injury. Journal of neuroinflammation. 2013; 10: 871.

6. Maqbool M, Vidyadaran S, George E, et al. Human mesenchymal stem cells protect neutrophils from serum-deprived cell death. Cell biology international. 2011; 35: 1247-1251.

7. Chamberlain G, Fox J, Ashton B, et al. Concise review: mesenchymal stem cells: their phenotype, differentiation capacity, immunological features, and potential for homing. Stem cells. 2007; 25: 2739-2749.

8. Gupta N, Krasnodembskaya A, Kapetanaki M, et al. Mesenchymal stem cells enhance survival and bacterial clearance in murine Escherichia coli pneumonia. Thorax. 2012; thoraxjnl-2011-201176.

9. Mócsai A. Diverse novel functions of neutrophils in immunity, inflammation, and beyond. Journal of Experimental Medicine. 2013; 210: 1283-1299.

10. Brandau S, Jakob M, Hemeda $\mathrm{H}$, et al. Tissue-resident mesenchymal stem cells attract peripheral blood neutrophils and enhance their inflammatory activity in response to microbial challenge. Journal of leukocyte Biology. 2010; 88: 1005-1015.

11. Gupta N, Su X, Popov B, et al. Intrapulmonary delivery of bone marrow-derived mesenchymal stem cells improves survival and attenuates endotoxin-induced acute lung injury in mice. The Journal of Immunology. 2007; 179: 1855-1863.

12. Németh K, Leelahavanichkul A, Yuen PS, et al. Bone marrow stromal cells attenuate sepsis via prostaglandin E 2-dependent reprogramming of host macrophages to increase their interleukin-10 production. Nature medicine. 2009; 15: 42.

13. Ardeshir Abbasi SMAF, Norouz Delirezh Ali Mostafaei. Caffeine alters the effects of bone marrow-derived mesenchymal stem cells on neutrophils. Adv Clin Exp Med. 2018; 4: 6.

14. Pourtayeb S, Froushani SMA. Nicotine can modulate the effects of the mesenchymal stem cells on neutrophils. Advances in medical sciences. 2017; 62: 165-170.

15. Pålsson-McDermott EM, O’neill LA. Signal transduction by the lipopolysaccharide receptor, Toll-like receptor-4. Immunology. 2004; 113: 153-162.

16. Abbasi A, Kukia NR, Froushani SMA, et al. Nicotine and caffeine alter the effects of the LPS-primed mesenchymal stem cells on the cocultured neutrophils. Life sciences. 2018; 199: 41-47.

17. Cho DI, Kim MR, Jeong Hy, et al. Mesenchymal stem cells reciprocally regulate the M1/M2 balance in mouse bone marrow-derived macrophages. Experimental \& molecular medicine. 2014; 46: e70.

18. Le Blanc K, Mougiakakos D. Multipotent mesenchymal stromal cells and the innate immune system. Nature Reviews Immunology. 2012; $12: 383$.

19. Sukho P, Hesselink JW, Kops N, et al. Human mesenchymal stromal cell sheets induce macrophages predominantly to an anti-inflammatory phenotype. Stem cells and development. 2018.

20. Shushtari N, Froushani SMA. Caffeine Augments The Instruction of Anti-Inflammatory Macrophages by The Conditioned Medium of Mesenchymal Stem Cells. Cell Journal (Yakhteh). 2017; 19: 415.

21. Motlagh BM, Ahangaran NA, Froushani SMA. Calcitriol modulates the effects of bone marrow-derived mesenchymal stem cells on macrophage functions. Iranian journal of basic medical sciences. 2015; $18: 672$. 